\title{
PROGRESSIVE NEUROACANTHOCYTOSIS IN BROTHERS: A CASE REPORT
}

Shaheen E Lakhan, Kenneth Gross

\section{INTRODUCTION}

Choreoacanthocytosis, more broadly called neuroacanthocytosis, is a rare neurodegenerative disorder usually inherited as an autosomal recessive trait. It is one of the neuroacanthocytosis syndromes characterized by neurological problems and acanthocytosis. Symptoms typically become apparent between the ages of 25 and 45 . The disorder may be characterized by chorea, dystonia, a buckling gait, tics including lip "smacking" and "hissing" vocalizations, personality changes, cognitive decline, seizures, parkinsonism, and/or atrophy of muscle tissue (amyotrophy) with an abnormally high lengthwise arch of the foot (pes cavus). ${ }^{1,2}$ Early changes may include personality alterations, most often depression. At some point throughout the disease, the peripheral blood smear usually reveals acanthocytes-spiculated red cells with a few finger-like projections of variable size (width and length) and surface distribution.

\section{CASE DESCRIPTION}

A 46-year-old man of Puerto Rican descent presented with a worsening movement disorder. He had experienced a 10-year history of progressive orofacial dyskinesia, dysarthria, dysphagia, and chorea of the trunk and limbs. Six years previously, he had experienced generalized tonicclonic seizures.

At the time of admission, the patient was alert and cooperative, appeared to be comfortable, and had normal vital signs. Results of cardiac chest and abdominal examinations were normal. He had intermittent oral, facial, and neck movements and mimicked sucking and grimacing associated with intermittent rapid brief eye closings and occasional flexion of his neck. He made kissing and sucking sounds, but uttered no involuntary words or vocalizations. There was chorea of the trunk and, to a lesser extent, of

Global Neuroscience Initiative Foundation (GNIF)- Los Angels, CA, United States

Email: slakhan@gnif.org the limbs. His communicative speech had a pronounced nasal quality and was slurred, and the prosody of speech was irregular. With dentures, he was still unable to chew solid food and often regurgitated liquids from his nose while drinking.

All distal muscles of his 4 limbs were atrophic. His thumbs and fifth digits were foreshortened and his feet highly arched.

All cranial nerves (I-XII) were normal except for the orofacial movements, dysarthria, and dysphagia. The strength of all the muscles about the ankles, feet and, to a lesser extent, hands was diminished. Deep tendon reflexes and plantar responses were all absent. Sensation of position, vibration, light touch, and pinprick were intact. Ataxia of the limbs and trunk was not present, but skilled movements, sitting, and walking were unsteady and irregular because of the chorea.

In the genetic history, there was no consanguinity. The patient had 3 brothers and 1 sister. Only 1 sibling, an older brother, 51, had any medical or neurological history of note. This brother began to experience dysarthric speech, along

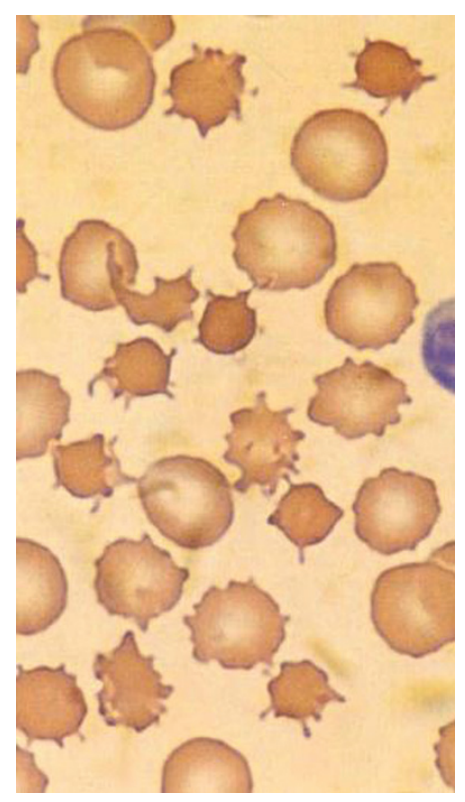

Figure 1 - The spiked red blood cells (acanthocytes) on the peripheral blood. 


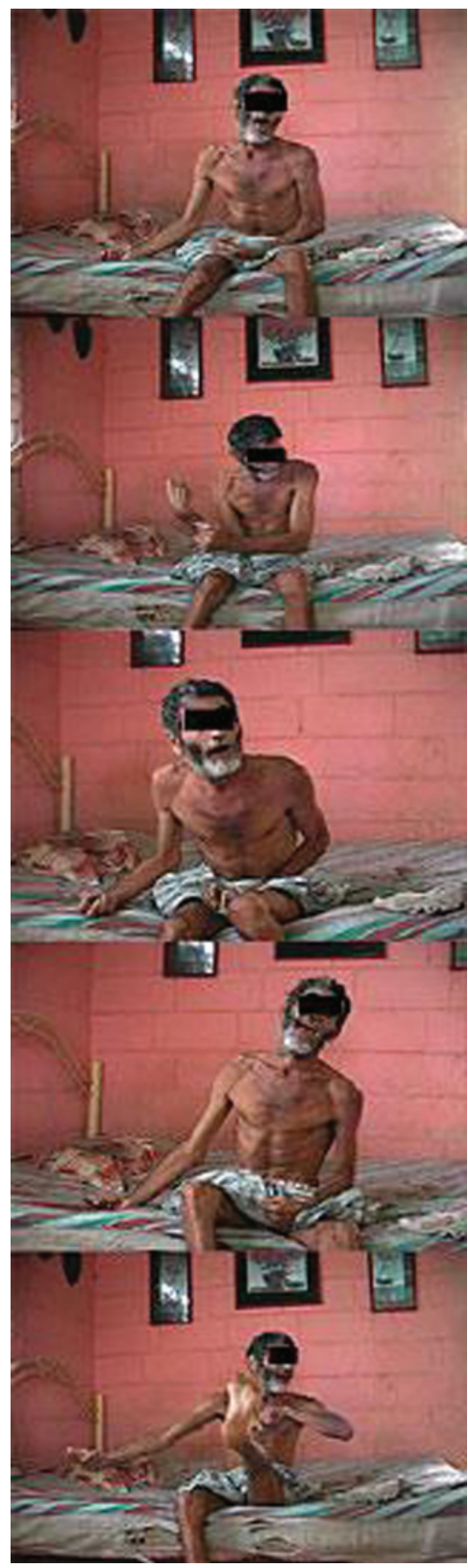

Figure 2 - Sequential head-neck-leg tic, dystonic/chorea movements. Muscle atrophy also noted.

with unsteadiness of gait and intermittent generalized tonicclonic seizures at the age of 40 . Limb atrophy was minimal, whereas the dysarthria was nearly as pronounced as in the patient; the choreiform movements and orofacial tics were only moderate in comparison.

The clinical workup for the patient was as follows: electroencephalograph (EEG) - paroxysmal slow wave activity, bilaterally and diffusely; head computed tomography (CT) and magnetic resonance imaging (MRI)—bilateral, mild caudate atrophy; electromyography (EMG)—hyperirritability and partial denervation of proximal and distal muscles-all limbs; nerve conduction velocities (NCV)—

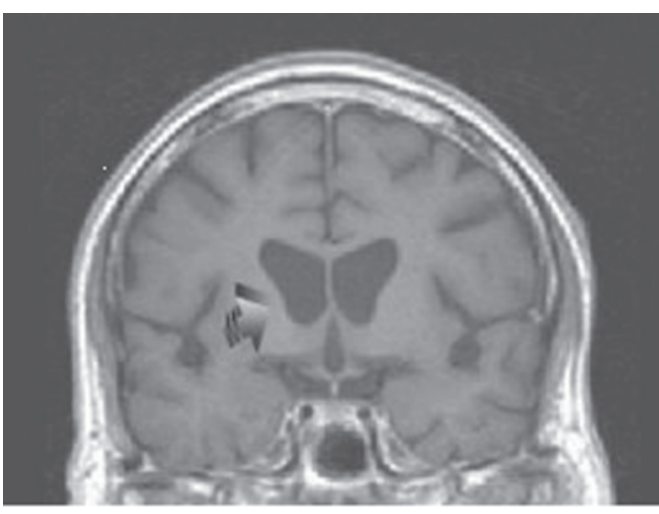

Figure 3 - Caudate atrophy on brain MRI.

normal; echocardiogram-hypertrophic cardiomyopathy; muscle biopsy-triceps revealed clusters of small, angulated fibers consistent with advanced denervation atrophy; blood tests-thyroid studies, collagen vascular serology, and lipids - all normal; Liver enzymes—normal; creatine phosphokinase (CPK)—400 (high) all muscle types (also noted in the affected brother); Blood group- $\mathrm{O}+$, no Kell null phenotype; Peripheral smear-20\% acanthocytes among otherwise normal-appearing RBC's (same pattern noted in the affected brother).

\section{DISCUSSION}

This cases raises several issues regarding the generation of a differential diagnosis and in the decision as to which tests to pursue to elucidate a unitary diagnosis.

The patient had such a complicated presentation that one must also ask: Is there a unitary diagnosis? Five critical areas should be considered as follows: 1) movement disorder with chorea; 2) movement disorder with tics; 3) seizures; 4) distal limb atrophy; and 5) genetics.

If one were to review all adult onset choreas, the leading candidate would be Huntington's disease (HD). The chorea in HD is not a single type. ${ }^{3}$ However, strong involvement of the limbs, as opposed to orofacial dyskinesia, and truncal chorea would be more typical of HD. Other causes of adult onset chorea include thyroid disease, lupus, druginduced, pregnancy, stroke, and an idiopathic type usually seen in older patients. Having noted all this, the bilaterality rules out stroke; the patient's sex, age, and drug history essentially rule out other causes; and we would not be faulted to check for a rare thyroid presentation of chorea or a rare lupus presentation.

Gilles de la Tourette syndrome (TS) would be dominated by tics, yet the patient's midlife age of onset also makes this diagnosis unlikely. ${ }^{4}$ Tardive dyskinesia might also be considered, but the absence of a psychotropic drug history rules it out. Lesch-Nyhan syndrome would have 
produced the facial movement problems in childhood, and the orofacial involuntary movements in Meige syndrome do not extend to the trunk, limbs, and gait.

Although TS and, particularly, HD (as an autosomal dominant disorder) can have the sibling connection seen here, 2 additional aspects argue against TS and HD. One is seizures; the other is limb atrophy. The seizures may have occurred as a result of head trauma induced by falls. Limb atrophy could also be part of a neuropathy, which can also have no defined cause. It is worthwhile, however, to assume that these aspects are part of the neurological syndrome because they are both noted in varying degrees in the affected brother.

Seizures, of course, can be of genetic origin when occurring in siblings. No cause is commonly found for inherited seizures that begin in adulthood. If one speaks of limb atrophy of genetic origin in adults, disorders such as Charcot-Marie-Tooth or one of the commonly inherited muscular dystrophies come to mind. Neither features chorea. $^{5}$

The job of the clinician at this point is to attempt to define all the above features more clearly in order to articulate a differential diagnosis. Through a process of elimination, it is also quite reasonable to move to a consideration of unusual conditions, including neuroacanthocytosis. This would not be "hunting zebras," because the entire clinical presentation so far makes several rare disorders and odd presentations of more common diseases unlikely. In reference to this latter point, both lupus and thyroid disease can induce chorea, seizures, and neuropathy. However, either features orofacial tics nor a striking genetic link in the family tree.

The caudate atrophy noted on the CT/MRI would be typical of HD or other degenerative disorders involving chorea ${ }^{6}$ However, one laboratory test result not noted in HD must be the subject of great focus in elucidating this case, ie, the presence of acanthocytosis on the peripheral smear. Acanthocytosis can occur in the presence of liver disease or in lipid abnormalities, such as Bassen-Kornzweig syndrome, a genetic disease of childhood, which shows ataxia, diarrhea, and abetalipoproteinemia. No evidence for a liver disorder or a childhood lipid disorder was noted in this case.

An axonal neuropathy combined with an elevated CPK is not typical of a disorder such as HD or TS. Huntington's disease can show a raised CPK related to the movement disorder, which may be the reason for the elevation here.

In a rare X-linked disorder called McLeod syndrome, there can be an elevated CPK as well as skeletal muscle wasting with chorea and acanthocytosis. ${ }^{7,8}$ This is associ- ated with the Kell null blood group phenotype, which was not present in this patient, however. ${ }^{9}$

A cardiomyopathy is commonly described in McLeod syndrome, but not in HD. ${ }^{8}$

The genetic disorder suggested here would be the autosomal recessive disorder called choreoacanthocytosis (also termed Levine-Critchley syndrome), which can include chorea, tics, neuropathy/amyotrophy, seizures, and cardiomyopathy due to an abnormality on chromosome $9 .{ }^{10}$ The chorea, acanthocytosis, and hyperCKemia in both the patient and his brother clinch this diagnosis.

Another neuroacanthocytosis syndrome, Huntington disease-like 2 (HDL2) is an autosomal dominant disorder associated with CAG/CTG trinucleotide repeats on chromosome 16 (not the Huntington gene). ${ }^{11}$ Huntington diseaselike 2 has onset in the fourth decade with a similar description to that of choreoacanthocytosis (ie, choreoathetosis, dystonia, parkinsonism); however, it does not involve orofacial dyskinesias, neuropathy, and cardiomyopathy. Moreover, HDL2 typically features weight loss and hyperreflexia, both of which were not noted in this patient.

Unfortunately, this patient and his brother had typical progressive courses of neuroacanthocytosis that involved worsening clinical depression and muscle wasting. Advancing dysphagia lead to their deaths due to aspiration pneumonia after about 15 years of the disease.

Autopsy in these patients revealed features similar to what is noted in HD in the brain, ie, atrophy and astrocytic gliosis of the caudate, putamen, and globus pallidus. In contrast to HD, no cerebral atrophy existed, but diffuse gliosis in the white matter of the cerebral hemispheres and spinal cord gray matter occurred, along with patchy demyelination of peripheral nerves. ${ }^{12,13}$

\section{CONCLUSION}

This case illustrates that it is a worthy task to pursue a unitary diagnosis even in a complex case, particularly where there is a neurogenetic syndrome suggested. Neurology is replete with rare diseases and unusual presentations. Thinking within only 1 specialty is also a poor plan. As noted in this case, disease overlap exists-from the purely neurological aspects to pathology in the hematological and cardiac domains in the same patient. All features comprise a single syndrome.

It is also noteworthy to recall that neuroacanthocytosis has multiple neurological, cardiac, and hematological presentations, so that one sees unusual presentations within an already rare disease. The most common genetic pattern for the illness, which was born out in this patient and his brother, however, was the presence of a deletion mutation 
on the gene known as VPS13A localized to chromosome band $9 \mathrm{q} 21 .{ }^{14}$ This leads to a chorein protein deficiency. Like the defect in HD in which an abnormal protein called huntingtin is produced, the precise function of the implicated protein is not understood.
Most likely is the fact that this gene defect is responsible, at least in part, for a membrane problem that induces the acanthocytosis, along with cardiac, skeletal muscle, anterior horn cell/nerve, and basal ganglia/cortical neuronal derangements.

\section{REFERENCES}

1. Critchley EM, Clark DB, Wikler A. Acanthocytosis and neurological disorder without abetalipoproteinemia. Arch Neurol. 1968;18:134-40.

2. Hardie RJ, Pullon HW, Harding AE, Owen JS, Pires M, Daniels GL, et al. Neuroacanthocytosis: A clinical, haematological and pathological study of 19 cases. Brain. 1991; 114 (Pt 1A):13-49.

3. Walker FO. Huntington's disease. Semin Neurol. 2007;27:143-50.

4. Shavitt RG, Hounie AG, Rosario Campos MC, Miguel EC. Tourette's syndrome. Psychiatr Clin North Am. 2006;29:471-86.

5. Andersson PB, Rando TA. Neuromuscular disorders of childhood. Curr Opin Pediatr. 1999;11:497-503.

6. Kutcher JS, Kahn MJ, Andersson HC, Foundas AL. Neuroacanthocytosis masquerading as Huntington's disease: CT/MRI findings. J Neuroimaging. 1999;9:187-9.

7. Swash M, Schwartz MS, Carter ND, Heath R, Leak M, Rogers KL. Benign X-linked myopathy with acanthocytes (McLeod syndrome). Its relationship to X-linked muscular dystrophy. Brain. 1983; 106 (Pt 3):717-33.

8. Cavalli G, de Gregorio C, Nicosia S, Melluso C, Serra S. Cardiac involvement in familial amyotrophic chorea with acanthocytosis: description of two new clinical cases. Ann Ital Med Int. 1995;10:24952.
9. Dotti MT, Battisti C, Malandrini A, Federico A, Rubio JP, Circiarello G et al. McLeod syndrome and neuroacanthocytosis with a novel mutation in the XK gene. Mov Disord. 2000;15:1282-4.

10. Walker RH, Danek A, Dobson-Stone C, Guerrini R, Jung HH, Lafontaine $\mathrm{AL}$, et al. Developments in neuroacanthocytosis: expanding the spectrum of choreatic syndromes. Mov Disord. 2006;21:1794-805.

11. Margolis RL, O'Hearn E, Rosenblatt A, Willour V, Holmes SE, Franz ML, et al. A disorder similar to Huntington's disease is associated with a novel CAG repeat expansion. Ann Neurol. 2001;50:373-80.

12. Bird TD, Cederbaum S, Valey RW, Stahl WL. Familial degeneration of the basal ganglia with acanthocytosis: a clinical, neuropathological, and neurochemical study. Ann Neurol. 1978;3:253-8.

13. Sobue G, Mukai E, Fujii K, Mitsuma T, Takahashi A. Peripheral nerve involvement in familial chorea-acanthocytosis. J Neurol Sci. 1986;76:347-56.

14. Danek A, Dobson-Stone C, Velayos-Baeza A: The phenotype of choreaacanthocytosis: a review of 106 patients with VPS13A mutations. Moy Disord. 2005;20:1678. 